\title{
Soziale Arbeit im Modus der Vermittlung
}

\author{
»Stellvertretende Krisenbewältigung « als Modell
}

Klaus Kraimer

Theorie und Praxis treten auf der Stelle, wenn sozialwissenschaftliche Modelle unterrepräsentiert sind, die zentrale Strukturprobleme der Sozialen Arbeit betreffen. Die Professionalisierungsbedürftigkeit Sozialer Arbeit als Dienstleistung in der Folge einer prinzipiellen Nicht-Standardisierbarkeit lässt sich mit Blick auf solche Modelle anspruchsvoll erläutern.

Dieses Monitoring richtet sich auf die Sozialisations- und Professionstheorie von Ulrich Oevermann. Von eminenter Bedeutung für die notwendige Befreiung von zu kurz greifenden Standardreaktionen in der Sozialen Arbeit sind in gleicher Weise die biografie- und professionstheoretischen Studien von Fritz Schütze, insbesondere zum biografischen Sprechen und zur Verlaufskurvenproblematik. Hier liegen eine Reihe von Untersuchungen vor, die unabdingbar sind für das Verständnis der Strukturprobleme in den Arbeitsabläufen zwischen Akteuren und Rat- und Hilfesuchenden in krisenhaften Lebenssituationen und Lebenslagen: Modelle wie »Stellvertretende Krisenbewältigung " (Oevermann) und »Paradoxien professionellen Handelns « (Schütze) sind prominente Beispiele.

Dem Prozess der Sozialisation standhalten - eine kontinuierlich zu bewältigende Anforderung. Verbunden mit zahlreichen Krisen in der Entwicklung, Persönlichkeitsbildung und Lebensbewäl-

Prof. Dr. Klaus Kraimer lehrt Theorie und Interventionslehre Sozialer Arbeit sowie Pädagogik an der Katholischen Hochschule für Soziale Arbeit in Saarbrücken und ist Privatdozent an der Universität Osnabrück.

E-Mail Klaus.Kraimer@t-online.de tigung fordert dies Soziale Arbeit als Profession und Disziplin heraus. Krisenhafte soziale Wandlungsprozesse bedingen »Bewährungsmodelle « angesichts veränderter Wissensbestände und Lebensbedingungen. Krisentypisches Wissen bedingt Modelle, die in Forschung, Ethik und in bewährten Strategien der Praxis gründen. Dies bedingt Vertrauen in das professionelle Handeln.

"Stellvertretende Krisenbewältigung " bezieht sich im »Modus der Vermittlung " (zwischen Theorie, Empirie und Praxis) auf Unterstützungsleistungen: Sowohl in Ablösungs- oder Entscheidungskrisen als
»Anamnese «, »Diagnose«, »Befund «, »Intervention « und "Evaluation «. Im Theorie-Modell sind diese Schritte (idealiter) vorgebildet und in der Profession in bewährter Weise als Handlungsmodelle verankert: Auf maieutische, also konstruktiv zu Erkenntnissen hinführte Weise wird beispielsweise in einer professionellen Praxis eruiert, welche Bedeutungen Klienten der eigenen Krisenbewältigung zuschreiben und welche Strategien verfolgt werden.

Vielfach ist es bereits hilfreich, diese durch »narratives Tun« zu unterstützen und in einer Atmosphäre der Beziehungs-

\section{»Autonomie der Lebenspraxis zu ermöglichen, ist der Prüfstein jeder Fremdhilfe»}

auch in ästhetischen, traumatischen Krisen oder in »Krisen durch Muße«. Als Kind, Jugendlicher, Erwachsener oder alter Mensch steht ein jeder, der nach Autonomie strebt, in solchen Krisen. Die Folge in der Spannung zwischen Wunsch und Wirklichkeit, Möglichkeit und Begrenztheit, Wollen und Sollen ist eine je individuelle Bewährungsdynamik, die es in krisenhaften Lagen zu rekonstruieren gilt, um eine Intervention zu begründen.

Sachlich folgt der Institutionalisierung des Lebens(ver)laufs - in dem Krisen entsprechend wirksam (inszeniert) werden die Institutionalisierung der Professionalisierung pädagogischer und sozialer Berufe. Deren Praxis zur stellvertretenden Autonomiebildung ist eine "klinische" und als sachlich erzwungene eine berufspraktisch abgekürzte Variante der Fallrekonstruktion (vgl. Oevermann 2000b, S. 59).

Zentrale Schaltstellen im Modus Operandi dieses Modells Sozialer Arbeit sind bereitschaft und des Vertrauens weiterzuentwickeln. "Narratives Tun " ist im Sinne der stellvertretenden Krisenbewältigung sowohl Handeln, als auch Erkennen, ZuErkennen und zu Erkennen-Geben. Autonomiebestrebungen - oft inadäquat ausgedrückt oder nicht erkannt - kann eine veränderte Bedeutung gegeben werden, in deren Begrifflichkeiten das krisenhafte der Situation >begreiflich $`$ wird.

Indem diese vorsichtig in Worte oder eine Form von Akzeptanz gefasst werden kann, lässt sich die Notwendigkeit der Stellvertretung für den Adressaten erkennen; eine mögliche Lösung kann erahnt, Vertrauen und Zuversicht können wachsen. Ein emotionales oder kognitives Ungleichgewicht lässt sich ausbalancieren, die Lebenssituation längerfristig stabilisieren. Eine solche Transformation ist entscheidend, weil sie erwartet wird, wenn auch zunächst nur durch ihre Erzählung in der Stellvertretung durch Intervention. 
Konstitutiv ist die Verpflichtung, das berufliche Engagement zwischen den Polen von "Routine " und "Krise " auf die Förderung der »Autonomie der Lebenspraxis « zu richten (in Betreuung, Pflege, Hilfe, Erziehung, Sozialisation, Bildung). Sowohl die krisenhafte Lebenspraxis selbst als auch die ihr entsprechende Professionspraxis ist als gemeinsamer Prozess (der Krisenbewältigung) zu lesen: Hier ist die Disziplin der wissenschaftlichen, die Profession der praktischen Begründung des Modells verpflichtet.

In der Theoriebildung (vgl. Oevermann 2004 S. 165 ff.) liegen ausgearbeitete Entwürfe als Krisentypen vor, die sich auf die verschiedenen Ebenen der traumatischen Krisen, die durch »brute facts « herbeigeführt werden, Entscheidungskrisen, die durch Optionen in der Lebenspraxis bezogen auf Sexualität, Leistung und Beruf sowie auf Staatsbürgerschaft entstehen sowie auf Krisen durch Muße (subjektiv herbeigeführt) bezogen sind. Insgesamt ist der Prozess der Konstitution von Erfahrung selbst in sich krisenhaft, da diesem die »systematische Erzeugung von Neuem « (ebd.) innewohnt.

Zwei systematische Gründe stehen der autonomen Krisenbewältigung entgegen (vgl. Oevermann 2002):

- manifeste Krankheit

- die Angewiesenheit auf Expertenleistungen angesichts des Fortschritts der methodisierten Wissensbestände durch Dienstleistungen

Als professionalisierungsbedürftige Expertenleistung bezieht sich Soziale Arbeit per Habitusbildung mit der notwendigen "Einsenkung « des Modells der stellvertretenden Krisenbewältigung in das Bewusstsein ihrer professionellen Praxis auf drei verschiedene, einander komplementäre Aufgabenstellungen (vgl. Oevermann 2000a, S. 67) im Kontext typischer - hier nur angedeuteter - Krisenszenarien (vgl. Oevermann 2004).

Modi stellvertretender Krisenbewältigung:

\section{Literatur}

Allert, T. (2007): Objektive Hermeneutik und fallrekonstruktive Forschung. Potenziale der soziologischen Perspektive für die klinische Forschung. In: Schaeffer, D./Müller-Mundt, G. (Hg.): Qualitative Gesundheits- und Pflegeforschung. Bern u. a., S. 103-118.

Mollenhauer, K. (1983): Vergessene Zusammenhänge. Über Kultur und Erziehung. München.

Oevermann, U. (2000a): Dienstleistungen der Sozialbürokratie aus professionalisierungstheoretischer Sicht. In: v. Harrach, E.-M. u. a. (Hg.): Verwaltung des Sozialen. Formen der subjektiven Bewältigung eines Strukturkonfliktes. Konstanz, S. 57-77.

Oevermann, U. (2000b): Die Methode der Fallrekonstruktion in der Grundlagenforschung sowie der klinischen und soziologischen Praxis. In: Kraimer, K. (Hg.): Die Fallrekonstruktion. Sinnverstehen in der sozialwissenschaftlichen Forschung. Frankfurt am Main, S. 58-156.

Oevermann, U. (2002): Professionalisierungsbedürftigkeit und Professionalisiertheit pädagogischen Handelns. In: Kraul, M. u. a. (Hg.): Biographie und Profession. Bad Heilbrunn/Obb., S. 19-63.

Oevermann, Ulrich (2004): Sozialisation als Prozess der Krisenbewältigung. In: Geulen, D./Veith, H. (Hg.): Sozialisationstheorie interdisziplinär. Stuttgart, S. 155-181.

Schütze, F. (1994): Das Paradoxe in Felix 'Leben als Ausdruck eines »wilden « Wandlungsprozesses. In: Koller, H. C./Kokemohr, R. (Hg.): Biographie als Text. Weinheim, S. 13-60.

Schütze, F. (2002): Supervision als ethischer Diskurs. In: Kraul, M. u. a. (Hg.): Biographie und Profession. Bad Heilbrunn/Obb., S. 135-164.

Stummbaum, M. (2007): Großer Aufholbedarf. Zum Verhältnis von sozialer Selbsthilfe und Sozialer Arbeit. In: Blätter der Wohlfahrtspflege 6, S. 230 f.

- Aufrechterhaltung und Erzeugung somato-psycho-sozialer Integrität: Therapie

- Erzeugung und Aufrechterhaltung von Gerechtigkeit: Rechtspflege

- Erzeugung und Aufrechterhaltung von methodisierter Geltung von Wissen und Normen

Szenarien typischer Ablösungskrisen:

- Krise der Geburt

- Ablösung von der frühkindlichen Mutter-Kind-Symbiose

- Ablösung von der Alleinzuständigkeit der Herkunftsfamilie/Adoleszenzkrise Eine Bewältigung der typischen Ablösungskrisen verspricht die Option, ein Leben in (relativer) Autonomie führen zu können - stets begleitet von Krisenszenarien, die dem »sozialisatorischen Versprechen « bedingungsloser Vertrauensbildung und Verlässlichkeit - wie noch in den Ablösungskrisen gültig - nicht verpflichtet sind.

Das Leben »stört « natürlich ständig durch vielerlei Schranken. Als regulative Idee kann - im Modus der Vermittlung »Aufforderung zur Selbsttätigkeit « hier als strukturelles Äquivalent wirksam werden. Konstitutiv ist die Spannung zwischen dem »Tatsächlichen « und dem "Möglichen « in der Ablauflogik von »Krise « und »Routine «.

Stellvertretende Krisenbewältigung als »Fremdhilfe « richtet sich so auf »Weckung von Selbsthilfe « - beides unabdingbar in der Gewinnung von Autonomie. Nicht eine vorrangig auf Wirkung erpichte Tätigkeit (vgl. Mollenhauer 1983, 121), sondern die gespannte Aufmerksamkeit der professionellen Hilfe für die Differenz zwischen dem, "was ist «, und dem, "was sein könnte « ist entscheidend. Beispielsweise ist eine Organisation von sozialer Selbsthilfe und Sozialer Arbeit erschließbar (vgl. Stummbaum 2007) wenn es gilt, die Autonomie der Lebenspraxis (wieder) zu ermöglichen: eine Bewährungsprobe für jede Form der Fremdhilfe. 